\title{
Niveaux de résidus de pesticides organochlorés dans les produits du cacao en Côte d'Ivoire
}

\author{
Henri Marius Godi BIEGO ${ }^{1 *}$, Adama COULIBALY ${ }^{1}$, Mathias Kouame KOFFI ${ }^{1,2}$, \\ Olivier Kouamé CHATIRE ${ }^{1}$ et Luc Philippe KOUADIO ${ }^{3}$ \\ ${ }^{1}$ Laboratoire de Biochimie et Sciences des Aliments, UFR Biosciences, Université de Cocody-Abidjan, \\ 22 BP 582 Abidjan 22, Abidjan, Côte d'Ivoire. \\ ${ }^{2}$ Laboratoire Central pour l'hygiène alimentaire et Agro-industrie, LANADA, \\ Ministère de la production animale et des ressources halieutiques, 04 BP 612 Abidjan 04, Côte d'Ivoire. \\ ${ }^{3}$ Département de Santé Publique, d'Hydrologie et de Toxicologie, UFR des Sciences \\ Pharmaceutiques et Biologiques, Université de Cocody. BP V 34 Abidjan, Côte d'Ivoire. \\ *Auteur correspondant, E-mail: biegoh3@yahoo.fr, Tél. /Fax: (225) 212560 43, Mobile: (225) 07018836
}

\section{RESUME}

L'objectif de cette étude est de déterminer le contenu en résidus de quelques pesticides organochlorés du cacao masse, des tourteaux et du beurre de cacao. Pour se faire, 75 échantillons de fèves de cacao ont été prélevés auprès des planteurs et coopératives du département d'Agboville et des exportateurs des ports de San Pedro et d'Abidjan. Les concentrations en 18 pesticides organochlorés ont été déterminées au chromatographe en phase gazeuse de marque Agilent Technologies 6980N munie de deux détecteurs à capture d'électrons

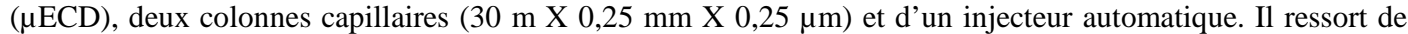
cette étude que 15 des 18 pesticides recherchés sont présents à des concentrations variant de $2 \mu \mathrm{g} / \mathrm{kg}$ à 59,6 $\mu \mathrm{g} / \mathrm{kg}$ dans les différents produits analysés. Les fèves de cacao issues du département d'Agboville se sont révélées plus contaminées en pesticides organochlorés (aldrine, alpha BHC, bêta BHC, delta BHC, lindane, dieldrine, endosulfane I, endosulfane II, endosulfane sulfate, endrine, endrine aldéhyde, endrine ketone, heptachlore, heptachlore epoxyde, 4,4'-DDD, 4,4'-DDE, 4,4'-DDTet metoxychlore), en nombre et en quantité. Il convient d'informer les différents acteurs du domaine d'activité sur les dangers encourus, d'éduquer les vendeurs et les planteurs et de contrôler leur activité par la mise en place de bonnes pratiques agricoles et le respect des normes sur les pesticides.

(C) 2009 International Formulae Group. All rights reserved.

Mots clés: pesticides organochlorés, produits du cacao, Chromatographie en Phase Gazeuse

\section{INTRODUCTION}

Le cacaoyer (Theobroma cacao linneaus, famille des Sterculiacées) est une plante stimulante des forêts tropicales. Le cacao représente $40 \%$ des recettes d'exportation de la Côte d'Ivoire avec en moyenne une production estimée à 1,4 millions de tonnes par an (Bonjean et Brun, 2008). Il est principalement cultivé de manière traditionnelle par des petits planteurs $(84 \%$ de la production) sur des exploitations inférieures à 5 ha (Fleischer et al., 1998).
L'utilisation de pesticides pour la lutte antivectorielle des nuisibles dans les plantations de cacao est une pratique relativement récente (Fleischer et al., 1998). Le gouvernement ivoirien a mis en place des textes réglementaires relatifs à la vente et l'utilisation de ces produits (Fleischer et al., 1998; DGAL, 2000; PNM, 2006). La Côte d'Ivoire a également adhéré aux accords internationaux sur les pesticides, notamment, le code de conduite de la FAO et le Principe d'Information et de Consentement Préalables 
(PNM, 2006). Cette utilisation a permis une amélioration des rendements agricoles. Cependant, elle suscite de nombreuses inquiétudes liées à la toxicité démontrée des substances chimiques utilisées ainsi qu'à leur influence négative sur l'environnement et la santé humaine. Ces composés sont, pour la plupart, lipophiles et s'accumulent dans les tissus de l'organisme. Au niveau aquatique, les pesticides entraîneraient des effets néfastes sur les organismes marins qui provoqueraient la disparition des phytoplanctons et zooplanctons ainsi que des poissons et crustacées (Bretaud, 2001; Benbakhta et al. 2007). Chez l'homme, ils seraient responsables des effets dermatologiques, mutagènes, cancérogènes, tératogènes, neurologiques et des troubles hormonaux (CSHPF, 1996; Repetto et Baliga, 1996; Daniels et al., 1997; Dewailly, 2000; Norström et al., 2000; Vine, 2001).

Ainsi, est-il probable de retrouver des résidus de pesticides dans les produits du cacao. Nous avons donc entrepris une étude exploratoire de détermination des résidus de pesticides organochlorés dans les produits issus du cacao.

\section{MATERIEL ET METHODES Echantillonnage}

Soixante quinze échantillons de fèves de cacao de $10 \mathrm{~kg}$ chacun ont été prélevés auprès des planteurs et coopératives du département d'Agboville et des exportateurs des ports d'Abidjan et de San Pedro sur une période de Janvier à Juin 2008. Au laboratoire, les concentrations en pesticides organochlorés ont été déterminés dans différents échantillons de cacao masse (75), de tourteaux (14) et de beurre de cacao avant (25) et après (25) filtration; soit au total 417 essais.

\section{Réactifs}

L'hexane $95 \% \quad$ (SDS) et L'eau désionisée (SDS) ont été utilisés. Un mélange de solution étalon comprenant 18 pesticides organochlorés (EPA 608 Supelco), à $20 \mu \mathrm{g} / \mathrm{l}$ a été utilisé. Il s'agit de l'aldrine, l'alpha BHC [cyclohexane, 1,2,3,4,5,6-hexachloro-, $(1 \alpha$, $2 \alpha, 3 \beta, 4 \alpha, 5 \beta, 6 \beta)]$, le bêta BHC [cyclohexane, 1,2,3,4,5,6-hexachlorure, $(1 \alpha$, $2 \beta, 3 \alpha, 4 \beta, 5 \alpha, 6 \beta)$ ], le delta BHC [cyclohexane, 1,2,3,4,5,6-hexachlorure, $(1 \alpha, 2 \alpha, 3 \alpha, 4 \beta, 5 \alpha$, $6 \beta)$, le lin-dane ou gamma BHC [cyclohexane,1,2,3,4,5,6-hexachlorure, $(1 \alpha$, $2 \alpha, 3 \beta, 4 \alpha, 5 \alpha, 6 \beta)]$, le dieldrine, l'endosulfane I, l'endosulfane II, l'endosulfane sulfate, l'endrine, l'endrine aldéhyde, l'endrine ketone, l'heptachlore, l'heptachlore epoxyde, le 4,4'DDD[benzene 1,1'-(2,2 dichloroethylidene) bis 4-chloro], le 4,4'-DDE[benzene 1,1'(dichloroethylidene) bis 4-chloro], le 4,4'DDT[benzene 1,1'-(2,2,2-trichloroethylidene) bis 4-chloro] et le metoxychlore.

\section{Appareillage}

L'appareil utilisé est un chromatographe en phase gazeuse (CPG) de marque Agilent Technologies 6890N, comprenant un injecteur automatique Agilent 7683, deux détecteurs à capture d'électron $(\mu E C D)$ et deux colonnes capillaires de marque Zebron (ZB-5MS et ZB-1701P; $30 \mathrm{~m} \mathrm{X} \mathrm{0,25} \mathrm{mm} \mathrm{X}$ $0,25 \mu \mathrm{m})$. L'appareillage est piloté par un micro-ordinateur muni d'un logiciel ChemStation plus version 2002. L'injection a été réalisée en mode Splitless et l'Azote N50 a été utilisé comme gaz vecteur.

\section{Conditions opératoires du chromatographe en phase gazeuse}

Les conditions opératoires du CPG ont été les suivantes: température de l'injecteur: $250{ }^{\circ} \mathrm{C}$; température des détecteurs: $310{ }^{\circ} \mathrm{C}$; volume injecté: $2 \mu \mathrm{l}$. La programmation des conditions analytiques du four est présentée dans le tableau 1.

\section{Extraction des pesticides Cacao masse}

Une prise d'essai de $500 \mathrm{~g}$ de chaque échantillon de fèves de cacao a été broyée au robot Moulinex. Après homogénéisation, $5 \mathrm{~g}$ de broyat ont été introduits dans un tube à centrifuger. $5 \mathrm{ml}$ d'hexane $95 \%$ et $15 \mathrm{ml}$ d'eau désionisée ont ensuite été ajoutés et le tube a été agité pendant 10 minutes au Top-Mix 11118; puis centrifugé pendant 10 minutes à une vitesse de rotation de $3500 \mathrm{tr} /$ minute au centrifugeur de marque Dynac. Après centrifugation, $2 \mathrm{ml}$ de surnageant ont été filtrés sur une cartouche contenant $300 \mathrm{mg}$ de florosil (Alltech) puis élué avec $2 \mathrm{ml}$ d'un mélange hexane-dichlorométhane (v/v; 85:15 $\mathrm{ml}$ ). La solution obtenue a été concentrée à 1 $\mathrm{ml}$ dans un tube à chromatographie, à l'aide 
Tableau 1: programmation des conditions analytiques du four.

\begin{tabular}{lcccc}
\hline \multicolumn{1}{c}{ Oven ramp } & ${ }^{\circ} \mathbf{C}$ /minute & Next $^{\circ} \mathbf{C}$ & Hold minute & Run time \\
\hline Initial & - & 80 & 0,5 & 0,50 \\
Ramp 1 & 30 & 175 & 0,0 & 3,67 \\
Ramp 2 & 1,5 & 200 & 0,0 & 20,3 \\
Ramp 3 & 3,5 & 280 & 4,0 & 47,2 \\
Ramp 4 & 40 & 300 & 10 & 57,7 \\
Post run & - & 60 & - & 57,7 \\
\hline
\end{tabular}

d'azote et conservée au réfrigérateur avant analyse. Trois essais ont été réalisés par échantillons de fèves de cacao.

\section{Beurre de cacao}

Une prise d'essai de $500 \mathrm{~g}$ de fèves a été broyée. Le cacao masse obtenu a été introduit par petite quantité dans une presse, de marque KOMET CA59 1H, pour l'extraction du beurre de cacao. La moitié du beurre obtenu a été filtrée à chaud à $60{ }^{\circ} \mathrm{C}$. Puis $5 \mathrm{~g}$ de beurre filtré ou non ont été introduits dans un tube à centrifuger pour l'extraction des pesticides, comme décrit pour le cacao masse. Trois essais ont également été réalisés par échantillons de beurre.

\section{Méthode de validation}

La validation a été réalisée selon la méthode AFNOR (AFNOR, 1996; CEE, 2000) et comprend l'étude de la linéarité, répétabilité, reproductibilité et du rendement d'extraction. La linéarité des 18 composés recherchés a été testée entre 0 et $15 \mu \mathrm{g} / \mathrm{l}$ à l'aide de 4 points d'étalonnage $(0 \mu \mathrm{g} / \mathrm{l}, 5$ $\mu \mathrm{g} / \mathrm{kg}, 10 \mu \mathrm{g} / \mathrm{l}$ et $15 \mu \mathrm{g} / \mathrm{l})$. Les essais de répétabilité et de reproductibilité ont été réalisés avec le standard à une concentration de $5 \mu \mathrm{g} / \mathrm{l}$. Trente essais ont été réalisés pour la répétabilité et pour la reproductibilité. Des ajouts de $5 \mu \mathrm{g}$ de l'étalon ont été réalisés afin de déterminer le rendement de l'extraction des composés. Dix essais distincts ont été réalisés pour l'ajout dosé.

\section{Analyses statistiques}

Les moyennes des concentrations des différents composés ont été calculées avec leurs écart-types; les coefficients de variation ont ensuite été obtenus pour exprimer la répétabilité et la reproductibilité. Le carré du coefficient de corrélation de Pearson a été calculé pour apprécier la linéarité. La proportion de composés ajoutée et dosée a servi à exprimer le rendement d'extraction. Les valeurs médianes et le domaine de variation des concentrations des différents composés retrouvés ont été utilisés pour décrire le niveau de contamination des échantillons. La variabilité des concentrations des pesticides organochlorés totaux a été étudiée par une analyse de variance à deux facteurs (sites de prélèvement et nature des produits analysés) effectuée par la procédure ANOVA utilisant le logiciel SPSS 12. Les concentrations moyennes totales en pesticides ont été séparées par la méthode de la plus petite différence significative $(\mathrm{p}<5 \%)$.

\section{RESULTATS \\ Validation des techniques d'extraction et de dosage}

Les résultats des essais de validation sont présentés dans le Tableau 2. Le carré du coefficient de corrélation $\left(\mathrm{R}^{2}\right)$ varie de 0,993 à 0,999 pour les 18 composés recherchés. Les coefficients de variations déterminés pour la répétabilité sont compris entre $1,0 \%$ et $1,6 \%$ alors que ceux déterminés pour la reproductibilité sont compris entre $2,4 \%$ et $4,2 \%$. Ces résultats traduisent une stabilité et une précision satisfaisantes de la technique chromatographique en phase gazeuse qui a été utilisée pour la détermination des composés recherchés. Les rendements d'extraction sont compris entre $94 \%$ et $99 \%$ pour les 18 pesticides recherchés; ce qui traduit une fiabilité convenable de la technique d'analyse.

\section{Concentrations en pesticides}

Les concentrations en pesticides organochlorés, retrouvées dans les produits analysés, sont présentées dans les tableaux 3 et 4 et les pourcentages d'échantillons contaminés sur la figure 1. Parmi les 18 composés recherchés, 3 n'ont pas été détectés dans les différents échantillons de cacao. Il 
Tableau 2: Résultats des essais de validation analytique du dosage des pesticides organochlorés.

\begin{tabular}{|c|c|c|c|c|c|}
\hline \multirow{3}{*}{ Composés } & \multicolumn{2}{|c|}{ Linéarité } & \multicolumn{2}{|c|}{ Coefficient de variation } & \multirow{2}{*}{$\begin{array}{l}\text { Rendement } \\
\text { d'extraction }\end{array}$} \\
\hline & Equation des droites & Carré du coefficient & Répétabilité & Reproductibilité & \\
\hline & d'étalonnage & de corrélation $\left(R^{2}\right)$ & $(\% ; \mathrm{n}=10)$ & $(\% ; n=15)$ & $(\% ; n=10)$ \\
\hline Alpha BCH & $Y=717,86 x$ & 0,993 & 1,3 & 3,6 & 96,1 \\
\hline Lindane & $Y=706,19 x$ & 0,998 & 1,0 & 3,3 & 97,3 \\
\hline bêta BCH & $Y=355,26 x$ & 0,995 & 1,5 & 2,6 & 99,0 \\
\hline Heptachore & $\mathrm{Y}=855,17 \mathrm{x}$ & 0,999 & 1,2 & 2,6 & 94,9 \\
\hline Delta BCH & $\mathrm{Y}=719,80 \mathrm{x}$ & 0,994 & 1,4 & 3,1 & 96,0 \\
\hline Aldrine & $Y=774,19 x$ & 0,993 & 1,3 & 3,6 & 98,9 \\
\hline \begin{tabular}{|l|} 
Endosulfan2 \\
\end{tabular} & $\mathrm{Y}=711,19 \mathrm{x}$ & 0,997 & 1,5 & 2,4 & 96,6 \\
\hline Heptachore epoxyde & $Y=700,99 x$ & 0,998 & 1,6 & 3,0 & 97,2 \\
\hline Endosulfan1 & $Y=1408,2 x$ & 0,998 & 1,1 & 2,7 & 97,1 \\
\hline 44'DDE & $Y=636,28 x$ & 0,994 & 1,2 & 3,9 & 98,4 \\
\hline Dieldrine & $\mathrm{Y}=1,0013 \mathrm{x}$ & 0,999 & 1,0 & 3,3 & 96,0 \\
\hline Endrine & $\mathrm{Y}=1,0030 \mathrm{x}$ & 0,999 & 1,3 & 3,3 & 94,0 \\
\hline DDT & $Y=0,9983 x$ & 0,999 & 1,2 & 4,2 & 95,3 \\
\hline Endrine aldehyde & $Y=0,9995 x$ & 0,999 & 1,4 & 2,6 & 98,6 \\
\hline 44'DDD & $\mathrm{Y}=0,9981 \mathrm{x}$ & 0,999 & 1,2 & 3,9 & 98,4 \\
\hline Endosulfane sulfate & $Y=0,9992 x$ & 0,999 & 1,2 & 3,3 & 95,2 \\
\hline
\end{tabular}

s'agit du DDT, du méthoxychlore et de l'endrine ketone. Les 15 autres pesticides organochlorés c'est-à-dire le lindane et les isomères alpha, bêta et delta BHC, l'aldrine, l'heptachlore et son isomère heptachlore époxyde, le DDD et son isomère DDE, l'endrine et son isomère l'endrine aldéhyde, le dieldrine et les endosulfane I, II et sulfate ont été retrouvés.

Les concentrations en pesticides organochlorés mesurées sont comprises entre $2 \mu \mathrm{g} / \mathrm{kg}$ et $59,6 \mu \mathrm{g} / \mathrm{kg}$ de produit. Les échantillons de cacao masse contiennent les concentrations en pesticides les plus élevées quel que soit le site de prélèvement (Tableau 4). Tous les échantillons de cacao masse de la région d'Agboville contiennent au moins un des pesticides analysés alors que seulement $58 \%$ des échantillons du port d'Abidjan et $35 \%$ de ceux de San Pedro sont contaminés (Figure 1). Il en est de même pour les échantillons de beurre filtré ou non. Les chantillons les plus contaminés proviennent de la région d'Agboville (Figure 1).

Ainsi, pour les échantillons prélevés au port d'Abidjan, les concentrations totales retrouvées sont de $18,2 \mu \mathrm{g} / \mathrm{kg}$ de cacao masse, $3,5 \mu \mathrm{g} / \mathrm{kg}$ de beurre non filtré et $2 \mu \mathrm{g} / \mathrm{kg}$ de beurre filtré. Pour la région d'Agboville, ces concentrations sont respectivement de 214,5 $\mu \mathrm{g} / \mathrm{kg}$ de cacao masse, $50,8 \mu \mathrm{g} / \mathrm{kg}$ de beurre non filtré et $2 \mu \mathrm{g} / \mathrm{kg}$ de beurre filtré. Enfin pour les échantillons prélevés au port de San Pedro, les concentrations retrouvées sont de $11,6 \mu \mathrm{g} / \mathrm{kg}$ de cacao masse, $8,9 \mu \mathrm{g} / \mathrm{kg}$ de beurre non filtré et $4,1 \mu \mathrm{g} / \mathrm{kg}$ de beurre filtré. L'homogénéité des concentrations en pesticides étudiée par une analyse de variance à deux facteurs (sites de prélèvement et nature des produits analysés), à travers le test $\mathrm{F}$, a révélé une différence significative au risque $\mathrm{p}<0,05$ entre les sites de prélèvement et la nature des produits analysés (Tableau 4).

\section{DISCUSSION}

L'étude réalisée révèle la présence de pesticides organochlorés variable en nombre et en quantité selon les sites de prélèvement et la nature des produits analysés. Cette variabilité pourrait s'expliquer par l'origine et la quantité des fèves de cacao brassées au niveau des différents sites. En effet, les ports 


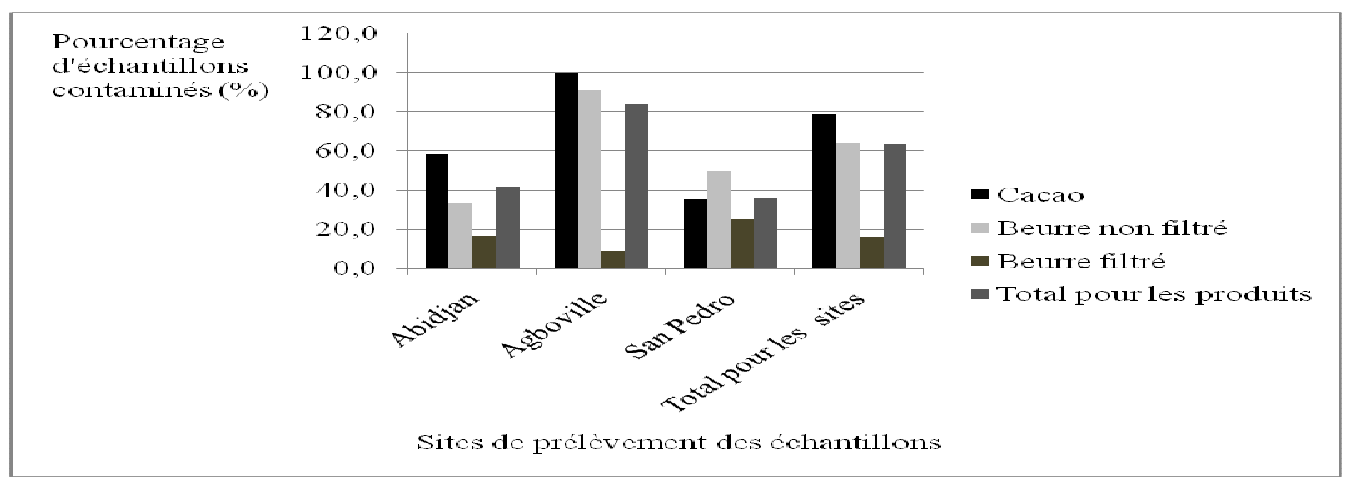

Figure 1: pourcentage d'échantillons contenant au moins un pesticide recherché en fonction des sites de prélèvement.

Tableau 3: valeurs médianes et [domaine de variation] des concentrations en pesticides organochlorés mesurées dans des échantillons de cacao (Concentration en $\mu \mathrm{g} / \mathrm{kg}$ de produit).

\begin{tabular}{|c|c|c|c|c|c|c|c|c|c|c|}
\hline & \multirow{2}{*}{\begin{tabular}{|l|} 
Oigine \\
Patie \\
anlycée \\
\end{tabular}} & \multicolumn{3}{|c|}{ Aligan } & \multicolumn{3}{|c|}{ Agboville } & \multicolumn{3}{|c|}{ San pedio } \\
\hline & & $\begin{array}{l}\text { Gacao } \\
\text { nasse }\end{array}$ & $\begin{array}{c}\text { Bume } \\
\text { nonfiltré }\end{array}$ & $\begin{array}{l}\text { Bume } \\
\text { filthé }\end{array}$ & $\begin{array}{l}\text { Cacao } \\
\text { masse }\end{array}$ & $\begin{array}{c}\text { Bume } \\
\text { monfiltúé }\end{array}$ & $\begin{array}{l}\text { Bume } \\
\text { fittré }\end{array}$ & $\begin{array}{l}\text { Gaco } \\
\text { masse }\end{array}$ & $\begin{array}{l}\text { Bume } \\
\text { monfiltú }\end{array}$ & $\begin{array}{l}\text { Bame } \\
\text { filtué }\end{array}$ \\
\hline \multirow{4}{*}{ HCH } & Apha & $\begin{array}{c}\mathbf{6 3} \\
{[3,87,9]}\end{array}$ & & & $\begin{array}{c}\mathbf{B} \\
{[8415,8]}\end{array}$ & $\begin{array}{c}\mathbf{6} \\
{[5,413,5]}\end{array}$ & & & & \\
\hline & Beta & & & & $\begin{array}{c}\mathbf{3 2 7} \\
{[109,936,0]}\end{array}$ & & & & & \\
\hline & Delta & & & & $\begin{array}{c}\mathbf{2} \\
{[13,640,0]}\end{array}$ & & & & & \\
\hline & Lindane & $\begin{array}{c}\mathbf{2 6} \\
{[2,1-4,2]}\end{array}$ & & & $\begin{array}{c}\mathbf{1 7 5} \\
{[10,822,6}\end{array}$ & $\begin{array}{c}\mathbf{5 4} \\
47-107] \\
\end{array}$ & & $\begin{array}{c}\mathbf{3 9} \\
{[3,64,2]}\end{array}$ & & \\
\hline \multicolumn{2}{|l|}{ Addine } & $\begin{array}{c}\mathbf{2 1} \\
{[2021]}\end{array}$ & & & $\begin{array}{c}\mathbf{5 9 9} \\
{[5,7-6,0]}\end{array}$ & & & & & \\
\hline \multicolumn{2}{|l|}{ Hptadlore } & & & & $\begin{array}{c}335 \\
60,059,6\end{array}$ & $\begin{array}{c}\mathbf{1 5 2} \\
{[20-15,2}\end{array}$ & & & & \\
\hline \multicolumn{2}{|c|}{ Hptadhoré́pxyde } & & & & & & & & $\begin{array}{c}\mathbf{2 2} \\
26-3,8]\end{array}$ & \\
\hline \multirow{3}{*}{ Fndosulfane } & I & & & & $\begin{array}{c}14,5 \\
{[4,9-19,3]}\end{array}$ & $\begin{array}{c}\mathbf{5 3} \\
{[4,47,7]} \\
\end{array}$ & & & $\begin{array}{c}\mathbf{2 2} \\
203,2]\end{array}$ & \\
\hline & II & & & & $\begin{array}{c}\mathbf{1 2 5} \\
20-125\end{array}$ & & & & & \\
\hline & Silfate & & & & $\begin{array}{c}27 \\
{[19,430,0}\end{array}$ & & & & & \\
\hline \multicolumn{2}{|l|}{$4 \mathrm{DEE}$} & & & & $\begin{array}{c}\mathbf{7 3} \\
{[61-10,2} \\
\end{array}$ & $\begin{array}{c}\mathbf{6 , 2} \\
{[5,47,0]} \\
\end{array}$ & & & & \\
\hline \multicolumn{2}{|l|}{$4 \mathrm{DDD}$} & $\begin{array}{c}\mathbf{3} \\
{[203,0]}\end{array}$ & & & & & & $\begin{array}{c}\mathbf{3 3} \\
{[3.1-3,4}\end{array}$ & $\begin{array}{c}45 \\
{[4446}\end{array}$ & $\begin{array}{c}\mathbf{4 1} \\
{[2044]}\end{array}$ \\
\hline \multicolumn{2}{|l|}{ Dldhine } & & & & $\begin{array}{c}\mathbf{1 3 , 5} \\
{[65-263]}\end{array}$ & $\begin{array}{c}\mathbf{1 2 7} \\
{[5.1-146}\end{array}$ & & & & \\
\hline \multicolumn{2}{|l|}{ Fndine } & $\begin{array}{c}\mathbf{4 2} \\
{[2042]}\end{array}$ & \begin{tabular}{|c|}
$\mathbf{3 5}$ \\
{$[203,5]$} \\
\end{tabular} & $\begin{array}{c}\mathbf{2 0} \\
{[2022]}\end{array}$ & $\begin{array}{c}\mathbf{1 0} \\
{[5,2-17,3]}\end{array}$ & & $\begin{array}{c}\mathbf{2 0} \\
{[2024]}\end{array}$ & $\begin{array}{c}\mathbf{4 4} \\
{[3,25,6]} \\
\end{array}$ & & \\
\hline \multicolumn{2}{|c|}{ Finchinealdhyde } & & & & $\begin{array}{c}\mathbf{5 1} \\
{[4,88,6}\end{array}$ & & & & & \\
\hline \multicolumn{2}{|c|}{ Cnnentrationtatale } & 182 & 3,5 & 20 & 2145 & 50,8 & 20 & 11,6 & 89 & 41 \\
\hline
\end{tabular}


Tableau 4: concentrations médianes totales en pesticides organochlorés retrouvées dans les différents produits analysés en fonction des sites de prélèvement (Concentration en $\mu \mathrm{g} / \mathrm{kg}$ de produit).

\begin{tabular}{|c|c|c|c|c|}
\hline$\overbrace{\text { Produits }}^{\begin{array}{c}\text { Site de } \\
\text { prélève ment }\end{array}}$ & A bidjan & Agboville & San Pedro & Moyenne \\
\hline Cacao masse & 18,2 & 214,5 & 11,6 & $81,4 \gamma$ \\
\hline Beurre non filtré & 3,5 & 50,8 & 8,9 & $21,6 \beta$ \\
\hline B eurre filtré & 2,0 & 2,0 & 4,1 & $2,7 \alpha$ \\
\hline Moyenne & 7,9 a & 89,1 b & $8,1 \mathrm{a}$ & 35,1 \\
\hline
\end{tabular}

Les concentrations totales moyennes suivies de mêmes lettres ou symboles ne sont pas significativement différentes au risque $\mathrm{p}<0,05$. Les lettres $\mathrm{a}$, b et $\mathrm{c}$ désignent une comparaison des sites d'échantillonnage. Les lettres $\alpha, \beta$ et $\gamma$ désignent une comparaison des produits analysés.

constituent des lieux de stockage, d'entreposage et de manutention du cacao des différentes régions de production avant l'exportation. Il y a donc une dilution des concentrations en pesticides mesurées dans les échantillons collectés dans les ports par rapport à celles retrouvées dans les échantillons provenant de la région de production d'Agboville. Des études réalisées dans la zone sud-ouest de la Côte d'Ivoire (première région de production du cacao et du café) ont mis en évidence la présence de pesticides organochlorés dans des échantillons de sols de trois plantations de cacaoyers (Karaki, 1997) et de poissons provenant du lac de Buyo (Traoré et al., 2003).

La présence de résidus de pesticides pose le problème de bonnes pratiques d'utilisation des pesticides dans la cacaoculture et de la mise en place du système de traçabilité au niveau de la filière cacao en Côte d'Ivoire (Bateman, 2008). Parmi les substances recherchées, seul le lindane a une Limite Maximale de Résidus (LMR), fixée à $1 \mathrm{mg} / \mathrm{kg}$ de produit (Codex Alimentarius, 1999). Cette LMR est remise en question à cause de la grande toxicité du composé. Ainsi, sa présence dans les échantillons de cacao des 3 sites de prélèvements; Abidjan $(2,6 \mu \mathrm{g} / \mathrm{kg}$ de cacao masse), Agboville $(17,5 \mu \mathrm{g} / \mathrm{kg}$ de cacao masse et $5,4 \mu \mathrm{g} / \mathrm{kg}$ de beurre non filtré) et San Pedro (3,9 $\mu \mathrm{g} / \mathrm{kg}$ de cacao masse) pourrait mettre en danger la santé du consommateur et constituer une source de pollution pour l'environnement (Sosan et al., 2008). Quant aux autres substances analysées, aucune d'entre elles ne bénéficie d'une homologation d'utilisation dans les plantations de cacao, à cause de leur degré de toxicité (Codex Alimentarius, 1999, 2005). Ainsi, ces substances ne doivent pas normalement être retrouvées dans des produits issus du cacao.

\section{Conclusion}

L'étude pilote réalisée a révélé la présence, à l'état de trace, de quelques pesticides organochlorés dans les échantillons de fèves de cacao collectés aux ports de San Pedro et Abidjan ainsi que dans la région d'Agboville. Parmi les 15 pesticides détectés 13 sont interdits d'utilisation en Côte d'Ivoire et seul un bénéficie d'une homologation conditionnelle. Les fèves de cacao sont relativement peu contaminées en pesticides organochlorés. Cependant, le caractère lipophile de ces composés, leur grande toxicité et l'absence de contrôles systématiques amènent à plus de vigilance afin de garantir une bonne qualité au cacao et protéger le consommateur contre les effets néfastes des pesticides sur la santé. Ainsi d'autres études devraient être entreprises pour établir une cartographie du niveau de résidus de pesticides organochlorés de la zone de production du cacao, poumon de l'économie ivoirienne.

\section{REFERENCES BIBLIOGRAPHIQUES}

AFNOR, 1996. Essai des eaux: protocole d'évaluation d'une méthode alternative d'analyse physico-chimique quantitative par rapport à une méthode de référence. 
In Association Française de Normalisation (éd). XP T ISSN 0335-3931: Paris; 210.

Bateman R, 2008. Pesticides use in cocoa: a guide for training, administrative and research staff. Report on consultations on the implementation of European Union legislation on Pesticide Residues (149/2008/EEC), London, p. 61.

Benbakhta B, Fekhaoui M, Abidi A, Adrissi L, Lecorre P. 2007. Résidus de pesticides organochlorés chez les bivalves et les poissons de la lagune de Moulay Bousselham Maroc. Afr. Sci., 3: 146-168.

Bonjean CA, Brun JF. 2008. Pouvoir de marché dans la filière cacao: hypothèse de Prebisch-Singer revisitée. Economie Prévision, 186: 133-144.

Bretaud S. 2001. Effets neurotoxiques des pesticides chez le carassin doré (carassius auratus). Thèse universitaire, Université de Rennes, p.167.

CEE (Communauté Economique Européenne). 2000. Quality Control Procedures or Pesticide Residue Analysis:Guidelines for residues Monitoring in the European Union (2 $2^{\text {nd }} e$ dn), N'SANCO/3103/2000; 30.

Codex Alimentarius. 1999. Le rapport de la trente et unième session du Comité du Codex sur les résidus de pesticides (CCPR). La Haye, p 90.

Codex Alimentarius. 2005. Residues in Food. Joint FAO/WHO Food Standard Program: Rome; 61-81.

CSHPF (Conseil Supérieur de l'Hygiène Publique de France). 1996. Rapport sur la fixation de limites maximales de résidus de produits phytosanitaires dans les aliments pour nourrissons et enfants en bas âge. CSHPF, p 32.

Daniels JL, Olshan AF, Savitz DA. 1997. Pesticides and childhood cancers. Environ. Health Perspect., 105: 10681077.

Dewailly E. 2000. Susceptibility to infections and immune Status in inuits infants exposed to organochlorines, Environ. Health Perspect., 108 : 205-211

DGAL (Direction Générale de l'Alimentation). 2000. Autorisation de mise sur le marché des produits phytosanitaires: guide des procédures. DGAL, Institut
National de Recherche Agronomique (INRA), Paris, p.97.

Fleischer G, Andoli V, Coulibaly M, Randolph T. 1998. Analyse socio économique de la filière des pesticides en Côte d'Ivoire. Série de Publication du Projet de Politique des Pesticides $\mathrm{N}^{\circ}$ 06/F, Novembre 1998. Institut des Sciences Economiques, Faculté d'Horticulture, Université de Hanovre, p 112.

Karaki H. 1997. Contribution à l'évaluation du niveau de contamination par les résidus organochlorés dans le sol de la région de Buyo: cas de trois plantations périphériques du lac. Mémoire du Diplôme d'Etudes Approfondis en Sciences de l'Environnement, Université d'Abobo-Adjamé, Abidjan, p.30.

Nordström M, Hadell L, Lindstrom G, Wingfors H, Hardell K, Linde A. 2000. Concentrations of organochlorines related to titers to epstein-barr virus early antigen $\mathrm{IgG}$ as risk factors for hairy cell leukemia, Environ. Health Perspect., 108 : 441-445.

PNM, 2006. Plan national de mise en œuvre de la convention de Stockholm sur les polluants organiques persistants. Ministère de l'Environnement, des Eaux et Forêts, Abidjan, Côte d'Ivoire, p 147.

Repetto R, Baliga SS. 1996. Pesticides and the Immune System: the Public Health Risks. World Resources Institute: Washington DC; 5.

Sosan MB, Akingbohungbe AE, Ojo IAO, Durosinmi MA. 2008. Insecticide residues in the blood serum and domestic water source of cacao farmers in Southwestern Nigeria. Chemosphere, 72: 781-784

Traoré SK, Mamadou K, Dembele A, Lafrance P, Banton O, Houenou P. 2003. Etude comparative du niveau de résidus des pesticides organochlorés chez trois espèces de poissons du lac de Buyo (sudouest de la Côte d'Ivoire) et estimation du potentiel de risque pour la santé humaine. J. Soc. Ouest-Afr. Chim., 16: 137-152.

Vine MF. 2001. Plasma 1,1-dichloro-2,2-bis ( $p$-chlorophenyl) ethylene (DDE) in levels and immune response, Am. J. Epidemiol., 221: 53-6. 